\title{
Comparative Microarray Analysis of Gene Expression During Activation of Human Peripheral Blood T Cells and Leukemic Jurkat T Cells
}

\author{
Zhaosheng Lin, G. Chris Fillmore, Tae-Hyun Um, Kojo S. J. Elenitoba-Johnson, and \\ Megan S. Lim \\ Department of Pathology (GCF, T-HU, KSJE-J, MSL) and the ARUP Institute for Clinical and Experimental \\ Pathology (ZL, KSJE-J, MSL), University of Utah, Salt Lake City, Utah
}

\begin{abstract}
SUMMARY: Activation of $\mathrm{T}$ cells involves a complex cascade of signal transduction pathways linking T-cell receptor engagement at the cell membrane to the transcription of multiple genes within the nucleus. The T-cell leukemia-derived cell line Jurkat has generally been used as a model system for the activation of T cells. However, genome-wide comprehensive studies investigating the activation status, and thus the appropriateness, of this cell line for this purpose have not been performed. We sought to compare the transcriptional profiles of phenotypically purified human $\mathrm{CD}^{+}{ }^{+} \mathrm{T}$ cells with those of Jurkat $\mathrm{T}$ cells during T-cell activation, using cDNA microarrays containing 6912 genes. About 300 genes were up-regulated by more than 2-fold during activation of both peripheral blood (PB) T cells and Jurkat T cells. The number of down-regulated genes was significantly lower than that of up-regulated genes. Only 79 genes in PB T cells and 37 genes in Jurkat T cells were down-regulated by more than 2 -fold during activation. Comparison of gene expression during activation of Jurkat and PB T cells revealed a common set of genes that were up-regulated, such as Rho GTPase-activating protein 1, SKP2, CDC25A, T-cell specific transcription factor 7 , cytoskeletal proteins, and signaling molecules. Genes that were commonly down-regulated in both PB T cells and Jurkat T cells included CDK inhibitors (p16, p19, p27), proapoptotic caspases, and the transcription factors $c$-fos and jun- $B$. After activation, 71 genes in PB T cells and only 3 genes in Jurkat T cells were up-regulated 4-fold or more. Of these up-regulated genes and expressed sequence tags, 44 were constitutively expressed at high levels in nonactivated Jurkat cells. Quantitative real-time RT-PCR analysis confirmed our microarray data. Our findings indicate that although there is significant overlap in the activation-associated transcriptional profiles in PB T cells compared with Jurkat T cells, there is a subset of genes showing differential expression patterns during the activation of the two cell types. (Lab Invest 2003, 83:765-776).
\end{abstract}

$M$ any studies of T-cell activation have been performed using either the human leukemic Jurkat cell line (Black et al, 1997; Ghaffari-Tabrizi et al, 1999) or primary T cells (Cooper and Pellis, 1998; Ellisen et al, 2001). T-cell activation is associated with regulation of multiple intracellular signaling events, mediated by either protein tyrosine kinases and serine/threonine kinases (Rudd, 1999; Tsuchida et al, 1999). Several key processes have been well documented during T-cell activation, including the activation of phospholipase C- $\gamma$, which is required for TCR-dependent activation of IL-2 (Lindholm et al, 1999). Up-regulation of protein kinase $\mathrm{C}$, a downstream target of phospholipase C- $\gamma$ (Cooper and Pellis, 1998; Ghaffari-Tabrizi et al, 1999), results in the stimulation of AP-1 transcriptional activity, which activates the transcription of

\section{DOI: 10.1097/01.LAB.0000073130.58435.E5}

Received March 13, 2003.

This work was supported by the ARUP Institute for Clinical and Experimental Pathology and National Institutes of Health grant CA 83984-01 (to KSJ-EJ).

Address reprint requests to: Dr. M. S. Lim, Department of Pathology, University of Utah, 50 North Medical Drive, Room A565, Salt Lake City, Utah 84132. E-mail: Megan.lim@path.utah.edu
c-Jun-N-terminal kinase/stress-activated protein kinase (Ghaffari-Tabrizi et al, 1999). Other signaling molecules such as mitogen-activated/extracellular signal-regulated kinase and calcium ions are also involved in T-cell activation (Tsuchida et al, 1999).

Both the leukemic Jurkat T-cell line (Black et al, 1997; Ghaffari-Tabrizi et al, 1999; Tanaka et al, 1997; Whisler et al, 1994) and primary PB T cells (Cooper and Pellis, 1998; Ellisen et al, 2001; Whisler et al, 1993) are commonly used as a model for human T-cell activation. Genomic expression programs in primary PB T-cell activation have been reported (Diehn et al, 2002; Ellisen et al, 2001; Feske et al, 2001); however, no genomic-scale comparison has been performed to analyze the activation-induced gene expression profiles of these two $T$ cell types.

The use of cDNA microarray technology is an effective approach for analysis of gene expression profiles in T-cell activation (Ellisen et al, 2001; Teague et al, 1999). The technology is based on two-color fluorescence hybridization in which two cDNA populations (from test and reference samples) are labeled separately with red or green fluorochrome and are subsequently hybridized to a microarray containing thousands of predeposited cDNAs (Schena et al, 1995). The ratio of fluorescence intensity (red/green) reflects 
the relative expression level between the tested cDNA populations (such as the test sample and the control sample). The technology has been used in the analysis of gene expression patterns, signaling pathways, and identification of new functional genes on a genomic scale (Chtanova et al, 2001; Ellisen et al, 2001; Teague et al, 1999).

In this study we sought to compare the gene expression profiles of phenotypically purified human $\mathrm{CD}^{+}{ }^{+} \mathrm{T}$ cells with those of Jurkat $\mathrm{T}$ cells during PHA-induced activation. The results show that general patterns of gene expression profiles were similar during activation of Jurkat $T$ cells and peripheral blood (PB) T cells. However, a subset of genes showed differential expression with varying degrees of gene regulation between the Jurkat and $P B T$ cells. This is the first comparative analysis of transcription profiles on a genomic scale between primary $\mathrm{T}$ cells and leukemic Jurkat $\mathrm{T}$ cells during T-cell activation (Gonzales and Bowden, 2002; Rowan et al, 1995).

\section{Results and Discussion}

\section{Overall Gene Expression Patterns Between Jurkat and PB T Cells During Activation}

The baseline similarity of gene expression between Jurkat and PB T cells was evaluated by hybridizing cDNA from resting $\mathrm{CD}^{+} \mathrm{PB} \mathrm{T}$ cells with cDNA from nonactivated Jurkat $T$ cells. The correlation coefficient between gene expression profiles of the two $\mathrm{T}$ cell types was 0.674 (Fig. 1), indicating that the two $T$ cell types were significantly different. By contrast, cohybridization of PB T-cell mRNA with themselves revealed a correlation coefficient of 0.99 (data not shown).

To compare the transcriptional profiles of PB T cells and Jurkat $T$ cells during activation, Cy-5-cDNAs from either activated Jurkat or PB T cells were hybridized with Cy-3-cDNAs from their nonactivated counterparts. Genes that were expressed greater than 2-fold (2-fold higher than control) after activation were con- sidered overexpressed, whereas genes that were expressed less than 0.5 fold (2-fold lower than control) were considered underexpressed. After 24 hours of activation, approximately 300 genes $(4.3 \%$ of 6912 genes tested) were overexpressed in each $\mathrm{T}$ cell type (Table 1). The number of underexpressed genes was much lower than that of the overexpressed genes in both $\mathrm{T}$ cell types, with only 79 genes $(1.1 \%$ of 6912 genes tested) in PB T cells and 37 genes $(0.5 \%$ of 6912 genes tested) in Jurkat $T$ cells. The ratio of up-regulated genes, expressed sequence tags (ESTs), and unknown genes are also shown in Table 1; the accession numbers of these ESTs and unknown genes are listed in Tables 2 and 3 . A significant portion of the up-regulated genes represents ESTs and unknown genes, highlighting the limited knowledge we have regarding the genes involved in T-cell activation. In PB T cells, the majority of the 6912 tested genes were expressed at similar levels (between the upper and lower blue lines in Fig. 1) during activation. This result is consistent with other reports that resting $T$ cells are not in a quiescent stage; instead, there are many cellular transcriptional activities that are needed to maintain viability and mobility of unstimulated $T$ cells (Boise et al, 1995; Teague et al, 1999; Vella et al, 1998). Jurkat $T$ cells are derived from a T-precursor lymphoblastic lymphoma (Black et al, 1997). As a result, many of the genes were expected to be constitutively expressed before activation treatment; however, Jurkat $\mathrm{T}$ cells responded to activation treatment similar to resting $T$ cells, as reflected by the similar numbers of up-regulated genes in both $T$ cell types (Table 1).

\section{Functional Groups of Similarly Expressed Genes in Both Jurkat and PB T Cells During Activation}

Analysis of the 300 genes that were overexpressed in each cell type revealed that many were unknown genes and ESTs. This suggests the existence of many novel, as yet uncharacterized, genes that are involved

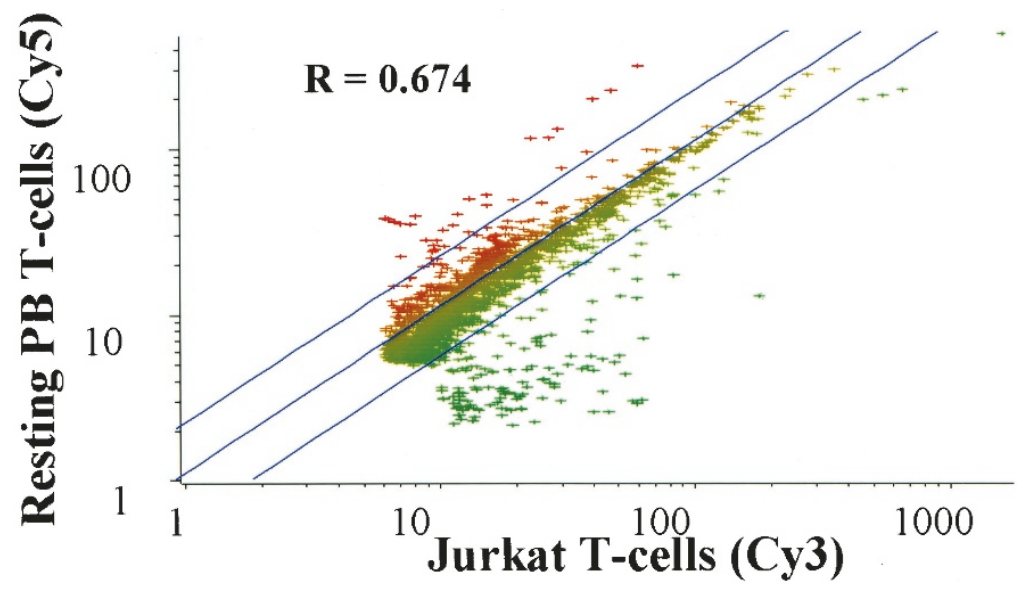

Figure 1.

Overall expression profile of 6912 genes between resting peripheral blood (PB) T cells and Jurkat T cells. Red and green data points indicate overexpressed and underexpressed genes, respectively. Genes above the upper blue lines represent those that were overexpressed by greater than 2-fold, whereas genes below the lower blue line represent those that were underexpressed by more than 2-fold. 
Table 1. Numbers of Differentially Expressed Genes During Activation of Jurkat and PB T Cells ${ }^{a}$

\begin{tabular}{lccc}
\hline & $\begin{array}{c}\geq 4 \text {-fold } \\
\text { up-regulated } \\
\text { Experiments (Cy5 vs Cy3) }\end{array}$ & $\begin{array}{c}\geq 2 \text {-fold } \\
\text { up-regulated }\end{array}$ & $\begin{array}{c}\geq 2 \text {-fold } \\
\text { down-regulated }\end{array}$ \\
\hline Activated PB T cells vs Resting PB T cells & $71 / 10: 4: 57$ & $300 / 76: 30: 194$ & $79 / 28: 34: 17$ \\
Activated Jurkat vs Jurkat T cells & $3 / 0: 0: 3$ & $301 / 62: 97: 142$ & $37 / 19: 8: 10$ \\
\hline
\end{tabular}

${ }^{a}$ Fold changes shown represent the ratio of gene expression in activated vs nonactivated cells.

Table 2. GenBank Accession Numbers of Overexpressed ESTs and Unknown Genes ( $\geq 2$-fold) During PB T-Cell Activation ${ }^{a}$

\begin{tabular}{|c|c|c|c|c|c|}
\hline 150221 & 23496 & 23157 & 17979 & 23384 & 19676 \\
\hline 249856 & 23492 & 20784 & 23964 & 23380 & 13016 \\
\hline 135527 & 9101 & 23162 & 15787 & 23375 & 13011 \\
\hline 233365 & 7863 & 23159 & 20563 & 23364 & 20025 \\
\hline 134753 & 23436 & 23245 & 22782 & 23363 & 21901 \\
\hline 135094 & 23477 & 23216 & 13404 & 23366 & 18363 \\
\hline 123065 & 23453 & 23256 & 20538 & 23365 & 23175 \\
\hline 123817 & 23691 & 23250 & 20512 & 22265 & 20032 \\
\hline 154789 & 11984 & 23207 & 9683 & 6660 & 12742 \\
\hline 295866 & 21464 & 21943 & 12406 & 21150 & 300237 \\
\hline 197637 & 21462 & 12602 & 20618 & 10586 & 23911 \\
\hline 194704 & 23635 & 20826 & 21755 & 22246 & 23405 \\
\hline 193617 & 20280 & 21978 & 22859 & 23388 & 12554 \\
\hline 246449 & 18764 & 23132 & 22815 & 23385 & 11690 \\
\hline 711857 & 15458 & 15871 & 23967 & 20236 & 23377 \\
\hline 141627 & 23618 & 10260 & 18197 & 7049 & 23419 \\
\hline 198694 & 11054 & 21845 & 17017 & 7039 & \\
\hline 121275 & 20373 & 17148 & 14557 & 21388 & \\
\hline 110582 & 20384 & 16911 & 15578 & 22495 & \\
\hline 110987 & 20382 & 23049 & 23714 & 21332 & \\
\hline 303048 & 8290 & 23031 & 21540 & 20228 & \\
\hline 206816 & 19958 & 23096 & 22688 & 19836 & \\
\hline 23616 & 19957 & 23059 & 23713 & 21173 & \\
\hline 23533 & 18832 & 7536 & 15521 & 23188 & \\
\hline 23114 & 19969 & 16099 & 16657 & 23181 & \\
\hline 22355 & 7053 & 20766 & 23707 & 23396 & \\
\hline 23398 & 23592 & 23122 & 23703 & 23393 & \\
\hline 21634 & 23590 & 23105 & 7275 & 23362 & \\
\hline 23121 & 9252 & 20716 & 20492 & 18493 & \\
\hline 23454 & 23598 & 12530 & 22701 & 18457 & \\
\hline 20394 & 23580 & 21893 & 1588 & 17373 & \\
\hline 19745 & 23536 & 23280 & 7268 & 23520 & \\
\hline 19739 & 23535 & 20981 & 21589 & 2355 & \\
\hline 19776 & 22419 & 23349 & 20419 & 23524 & \\
\hline 21269 & 23563 & 23358 & 18925 & 23523 & \\
\hline 13083 & 19873 & 10512 & 21597 & 17573 & \\
\hline 22388 & 12704 & 20952 & 23010 & 20150 & \\
\hline 21228 & 22162 & 20900 & 21790 & 19787 & \\
\hline 19734 & 20892 & 168 & 20620 & 2317 & \\
\hline 21232 & 20891 & 20916 & 20640 & 20178 & \\
\hline 20070 & 21003 & 66686 & 23007 & 22342 & \\
\hline
\end{tabular}

\footnotetext{
${ }^{a}$ This list was generated using GeneSpring software and the database available when this manuscript was prepared. Some of the genes listed are now identified known genes.
}

in T-cell activation. Activation of $\mathrm{T}$ cells leads to expression of multiple genes that are involved in cellular events such as proliferation, cytoskeletal construction, apoptosis, and secretion of cytokines (Ellisen et al, 2001; Teague et al, 1999). To determine the functional groups of genes that were commonly up- or down-regulated in T-cell activation, we examined the identities of 93 overexpressed and 15 underexpressed genes in both Jurkat and PB T cells. Of the genes that were up- and down-regulated by 1.5 fold or more, the 
Table 3. GenBank Accession Numbers of Overexpressed ESTs and Unknown Genes ( $\geq 2$-fold) During Jurkat T-Cell Activation $^{a}$

\begin{tabular}{|c|c|c|c|c|c|}
\hline 346134 & 293683 & 127185 & 23177 & 8745 & 23380 \\
\hline 233365 & 129567 & 120631 & 8556 & 20716 & 10586 \\
\hline 127408 & 129922 & 246143 & 21751 & 17148 & 22265 \\
\hline 294916 & 292171 & 196109 & 22843 & 16099 & 23477 \\
\hline 211202 & 144802 & 295492 & 21755 & 21887 & 23471 \\
\hline 346299 & 132323 & 295594 & 9683 & 18239 & 21228 \\
\hline 247901 & 128627 & 128118 & 510760 & 23096 & 9101 \\
\hline 245426 & 130801 & 20308 & 241874 & 23049 & 23465 \\
\hline 128735 & 138837 & 22594 & 17017 & 15871 & 19696 \\
\hline 246073 & 194656 & 21462 & 17803 & 10260 & 20025 \\
\hline 134753 & 234380 & 19945 & 7248 & 23188 & 7863 \\
\hline 154789 & 194401 & 21434 & 7298 & 23181 & 13963 \\
\hline 156023 & 194704 & 6957 & 17842 & 17265 & 66864 \\
\hline 193617 & 193533 & 23675 & 21505 & 972 & 23362 \\
\hline 155128 & 197637 & 19908 & 23713 & 23175 & 136801 \\
\hline 340840 & 195358 & 21408 & 23700 & 23137 & 138168 \\
\hline 67037 & 295866 & 18880 & 15578 & 23132 & 198961 \\
\hline 207379 & 142397 & 8292 & 23714 & 23162 & 127542 \\
\hline 21634 & 137760 & 16657 & 18132 & 23155 & 20280 \\
\hline 23121 & 137797 & 17740 & 23911 & 23023 & 23592 \\
\hline 144951 & 160730 & 8290 & 22806 & 21279 & 17373 \\
\hline 292452 & 244299 & 19958 & 15787 & 20116 & 18457 \\
\hline 203772 & 139189 & 19957 & 18109 & 20178 & 5546 \\
\hline 196125 & 248535 & 18832 & 18009 & 20156 & 23399 \\
\hline 209583 & 297102 & 21495 & 10043 & 21266 & 135094 \\
\hline 199327 & 247710 & 11984 & 17979 & 19734 & 247082 \\
\hline 196350 & 121521 & 21388 & 20584 & 19721 & 123817 \\
\hline 122161 & 38465 & 19836 & 23256 & 21253 & 127710 \\
\hline 23454 & 293417 & 19894 & 23246 & 19737 & 7053 \\
\hline 292207 & 110904 & 8173 & 22115 & 23562 & 23606 \\
\hline 198694 & 243770 & 9275 & 20892 & 23542 & 3210 \\
\hline 135527 & 110582 & 9255 & 15069 & 15521 & 23297 \\
\hline 276286 & 66390 & 9252 & 23222 & 23580 & 21173 \\
\hline 711857 & 245485 & 19824 & 8789 & 23536 & 20014 \\
\hline 128775 & 294133 & 9259 & 15068 & 11813 & \\
\hline 292679 & 206816 & 23641 & 23224 & 2317 & \\
\hline 196148 & 110987 & 15458 & 12742 & 23535 & \\
\hline 201207 & 122913 & 23660 & 20906 & 23523 & \\
\hline 66377 & 123196 & 23647 & 10512 & 21232 & \\
\hline 135212 & 122126 & 23618 & 20952 & 20007 & \\
\hline 135450 & 123065 & 18764 & 9984 & 21150 & \\
\hline
\end{tabular}

\footnotetext{
${ }^{a}$ This list was generated using GeneSpring software and the database available when this manuscript was prepared. Some of the genes listed are now identified known genes.
}

genes with known functions were grouped together (Tables 4 and 5). Among the known up-regulated genes (Table 4), several genes involved in cellular proliferation, cell adhesion, and cell cycle progression were identified, including CDC kinases, integrin, and Rho-activating protein-encoding genes (Klekotka et al, 2001; Lew and Reed, 1993; Welsh and Assoian, 2000). Genes encoding for protein synthesis machinery (eukaryotic translational termination factor 1 , ribosomal proteins L7a, L31, L21), cytoskeletal elements (myosin, actin, tubulin, calponin), and cell surface antigens (CD68, CD36, CD47) were also commonly up-regulated in both cell types during activation. Interestingly, a group of KIAA genes encoding proteins with potential signaling function, involved in cell migration (Chen et al, 2002) and metastasis (Lee et al, 2002), were also upregulated upon activation.

The down-regulated genes in both activated Jurkat and PB T cells consisted mostly of genes encoding CDK inhibitors, proapoptotic caspases, and transcription factors C-Fos and Jun-B (Table 5). Downregulation of CDK inhibitors is critical for inhibition of cell cycle progression. The stimulation of cell proliferation via PHA activation has been shown to induce the down-regulation of $\mathrm{p} 27^{\mathrm{Kip}}$ and $\mathrm{p} 21^{\mathrm{Waf}} \mathrm{CDK}$ inhibitors, resulting in the release of cells into G2/S phase of the cell cycle (Polyak et al, 1994; Sawada et al, 1996). The fact that some cytokines such as IL-4 (Boise et al, 1995; Vella et al, 1998) can only induce activated T 


\begin{tabular}{|c|c|c|c|c|}
\hline \multirow[b]{2}{*}{ Genban } & \multirow[b]{2}{*}{$\begin{array}{l}\text { Common } \\
\text { name }\end{array}$} & \multirow[b]{2}{*}{ Description } & \multicolumn{2}{|c|}{ Fold change in expression } \\
\hline & & & $\begin{array}{c}\text { Activated PB T } \\
\text { cells vs resting } \\
\text { PB T cells }\end{array}$ & $\begin{array}{l}\text { Activated Jurkat vs } \\
\text { nontreated Jurkat T } \\
\text { cells }\end{array}$ \\
\hline \multicolumn{5}{|c|}{ Proliferation/cell cycles } \\
\hline AA443506 & ARHGAP1 & Rho GTPase-activating protein 1 & 2.1 & 2.3 \\
\hline AA487634 & ARHGDIB & Rho GDP dissociation inhibitor (GDI) beta & 1.5 & 1.5 \\
\hline R22239 & SKP2 & S-phase kinase-associated protein 2 (p45) & 1.5 & 1.8 \\
\hline H15504 & ANXA7 & Annexin A7 & 1.5 & 1.9 \\
\hline R48796 & ITGAL & Integrin, alpha L [antigen CD11A (p180)] & 1.5 & 1.8 \\
\hline W44701 & IL3RA & Interleukin 3 receptor, alpha & 1.5 & 1.6 \\
\hline R09063 & CDC25A & Cell division cycle 25A & 3.6 & 1.6 \\
\hline H75547 & CLK1 & CDC-like kinase 1 & 1.5 & 1.9 \\
\hline AA459292 & CKS1 & CDC28 protein kinase 1 & 1.5 & 1.5 \\
\hline \multicolumn{5}{|l|}{ Antiapoptosis } \\
\hline \multirow{2}{*}{\multicolumn{5}{|c|}{$\begin{array}{l}\text { Transcription } \\
\text { factors }\end{array}$}} \\
\hline & & & & \\
\hline AA480071 & TCF7 & $\begin{array}{l}\text { Transcription factor } 7 \text { (T-cell specific, } \\
\text { HMG-box) }\end{array}$ & 1.5 & 2.0 \\
\hline \multirow{2}{*}{\multicolumn{5}{|c|}{ Protein synthesis }} \\
\hline & & & & \\
\hline AA496838 & RPL5 & Ribosomal protein L5 & 1.9 & 1.6 \\
\hline R01139 & RPL10A & Ribosomal protein L10a & 2.0 & 1.5 \\
\hline AA464034 & RPL21 & Ribosomal protein L21 & 3.2 & 1.7 \\
\hline H73727 & RPS14 & Ribosomal protein S14 & 3.6 & 1.8 \\
\hline \multicolumn{5}{|l|}{ Cytoskeleton } \\
\hline AA284568 & CNN2 & Calponin 2 & 1.7 & 2.1 \\
\hline AA488346 & MYL6 & Myosin, light polypeptide 6 & 2.1 & 1.7 \\
\hline W32281 & KRT8 & Keratin 8 & 1.5 & 1.9 \\
\hline T60048 & ACTG2 & Actin, gamma 2 & 1.7 & 1.6 \\
\hline AA180912 & TUBA1 & Tubulin, alpha 1 (testis specific) & 1.5 & 1.8 \\
\hline \multicolumn{5}{|c|}{ Proto-oncogenes } \\
\hline AA436591 & MERTK & c-mer proto-oncogene tyrosine kinase & 1.5 & 1.5 \\
\hline H90415 & BRCA1 & Breast cancer 1 , early onset & 1.5 & 2.0 \\
\hline \multicolumn{5}{|c|}{ Surface antigens } \\
\hline H69048 & CD36 & CD36 antigen (collagen type I receptor) & 1.5 & 1.6 \\
\hline AA455448 & CD47 & CD47 antigen (Rh-related antigen) & 2.1 & 2.1 \\
\hline AA421296 & CD68 & CD68 antigen & 2.3 & 2.6 \\
\hline AA486653 & CD81 & CD81 antigen & 1.9 & 1.6 \\
\hline \multicolumn{5}{|c|}{$\begin{array}{l}\text { Potential signaling } \\
\text { molecules }\end{array}$} \\
\hline H05563 & KIAA0182 & KIAA0182 protein & 1.6 & 2.3 \\
\hline AA456352 & KIAA0224 & KIAA0224 gene product & 1.6 & 1.6 \\
\hline N22435 & KIAA0229 & KIAA0229 protein & 1.7 & 1.6 \\
\hline T90374 & KIAA0798 & KIAA0798 gene product & 1.7 & 2.5 \\
\hline R91264 & KIAA0205 & KIAA0205 gene product & 1.7 & 2.0 \\
\hline N79669 & KIAA0231 & KIAA0231 protein & 1.7 & 2.0 \\
\hline AA486524 & KIAA0264 & KIAA0264 protein & 2.1 & 2.4 \\
\hline T74606 & KIAA0057 & KIAA0057 gene product & 1.8 & 2.3 \\
\hline
\end{tabular}

${ }^{a}$ Fold changes shown represent the ratio of gene expression in activated vs nonactivated cells.

cells (but not resting $T$ cells) to proliferate also supports these data.

Caspases are a group of proteases that are generally associated with apoptosis. Activation of several caspases including caspase- $1,-3,-8$, and -9 has been reported to induce T-cell apoptosis (MacFarlane et al,
2000; Morley et al, 2000; Ruiz et al, 2001; Takahashi et al, 2001; Varghese et al, 2001). Our data showed that several caspases (caspase-1, -3, -4, -7, -8, -9) were down-regulated in both Jurkat and PB T cells after 24 hours of activation (Table 5). This occurred during the period of T-cell proliferation in which cell numbers 
Table 5. Functional Groups of Genes That Were Down-Regulated During Activation of Both Jurkat and PB T Cells ${ }^{a}$

\begin{tabular}{ccc}
\hline & \multicolumn{2}{c}{ Fold change in expression } \\
\cline { 2 - 3 } Gene name & $\begin{array}{c}\text { Activated PB T cells } \\
\text { vs resting PB T cells }\end{array}$ & $\begin{array}{c}\text { Activated Jurkat vs } \\
\text { nontreated Jurkat T cells }\end{array}$ \\
\hline Cell growth and/or maintenance & & -3.0 \\
p16 & -2.2 & -2.5 \\
p19 & -2.1 & -2.1 \\
p27 & -1.8 & -2.4 \\
CDKI 3 & -2.2 & -2.6 \\
Death & & -1.9 \\
caspase 1 & -1.7 & -1.9 \\
caspase 3 & -1.5 & -2.4 \\
caspase 4 & -1.9 & -1.6 \\
caspase 7 & -1.5 & -2.5 \\
caspase 8 & -1.5 & -2.1 \\
caspase 9 & -1.9 & -1.9 \\
Transcription regulator & &
\end{tabular}

${ }^{a}$ Fold changes shown represent the ratio of gene expression in activated vs nonactivated cells.

increased by 3 -fold (data not shown). This is consistent with a previous report demonstrating that the activation of caspase 1 and 3 in $\mathrm{CD}^{+}$and $\mathrm{CD}^{+} \mathrm{T}$ cells was time dependent. The maximum activities of caspase-1 and -3 were observed after the proliferative period, at Days 7 and 10 (Ruiz et al, 2001).

The proto-oncogenes jun- $B$ and $c$-fos are generally involved in proliferation-associated transcriptional activation (Sylvester et al, 1998; Wang et al, 1997); thus, our results of their down-regulation were unexpected. Our results are, however, in keeping with those of Ellisen et al (2001) who observed down-regulation of jun- $B$ and $C$-fos in ConA-treated PB T cells. In our experiments, jun- $B$ and $c$-fos were down-regulated in both Jurkat and PB T cells during activation; this is consistent with other reports (Kreuzer et al, 1997; Passegue and Wagner, 2000) that C-Fos and Jun-B may be involved in the transcriptional activation of antiproliferative genes such as $p 16^{\mathrm{INK} 4 \mathrm{a}}$.

Diehn et al (2002) performed a study of gene expression profiles during PB T-cell activation. To compare our results with theirs, we used their web-based GeneXplorer software and Figure 1 (available at http:// genome-www.stanford.edu/costimulation) to search for the expression levels of 22 randomly selected genes from our list of genes that were up- or downregulated during $\mathrm{T}$-cell activation. The expression levels of these genes showed a high level of concordance between the two studies (18 of 22). Among the consistently expressed genes, there were proliferationrelated genes (four), CDK inhibitors (four), caspases (three), and transcription factors (two).

\section{Differences in Gene Expression Between Jurkat and PB T Cells During Activation}

Interestingly, many activation-induced genes in PB T cells were constitutively overexpressed to similar lev- els in the non-activated Jurkat T cells (Fig. 2, A and C). Of the 300 overexpressed (by 2 -fold) genes and ESTs, more than one-third (118 genes and ESTs) were expressed at similar levels in nonactivated Jurkat cells (Fig. 2A, Fig. 3). Although the vast majority of genes were similarly expressed between Jurkat and PB T cells during activation, one of the most significant differences between the expression profiles of the two cell types was the total number of genes that were expressed greater than 4-fold after activation (Table 1). Although 71 genes and ESTs were expressed greater than 4-fold in PB T cells, only three genes were overexpressed to this degree in Jurkat T cells. Among the $\sim 3002$-fold up-regulated genes during T-cell activation, there were only 93 genes (31\%) that were common between the two T cell types (Fig. 3).

Among the 71 genes and ESTs overexpressed by 4-fold or higher in activated PB T cells, more than half were constitutively overexpressed in nontreated Jurkat cells (Fig. 2C). These data suggest that when using Jurkat cells as model of T-cell activation, the number of activated genes may be underrepresented because many are constitutively overexpressed. The constitutively overexpressed genes (greater than 2-fold) in Jurkat cells (Table 6) most likely reflect the activated status of these leukemic $\mathrm{T}$ cells and probably are important in the pathogenesis of the neoplasm. Similar observations have been made when expression of phosphoinositide (PI)3-kinase was compared between PB T cells and Jurkat cells (Astoul et al, 2001). The finding that many of the constitutively overexpressed genes in Jurkat cells were also overexpressed in activated $\mathrm{PB} T$ cells suggests that genes induced during activation of $\mathrm{T}$ cells may be involved in leukemogenesis. Indeed, growth factors such as transforming growth factor- $\beta$ and TNF activate several signaling pathways including the PI3-kinase pathway (Kim et al, 2002) and the nuclear factor $-\kappa B$ pathway (Natoli et al, 
A. a

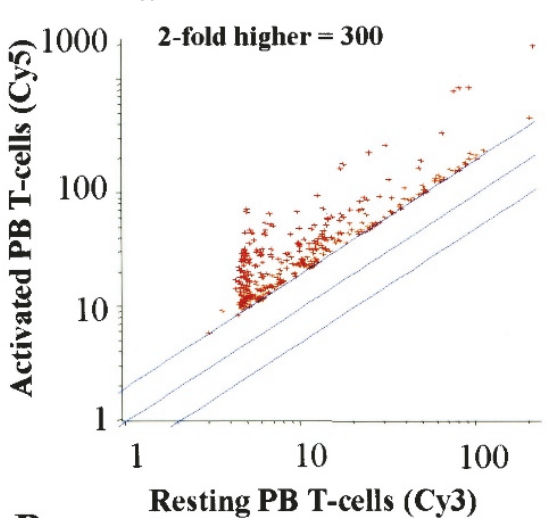

B.
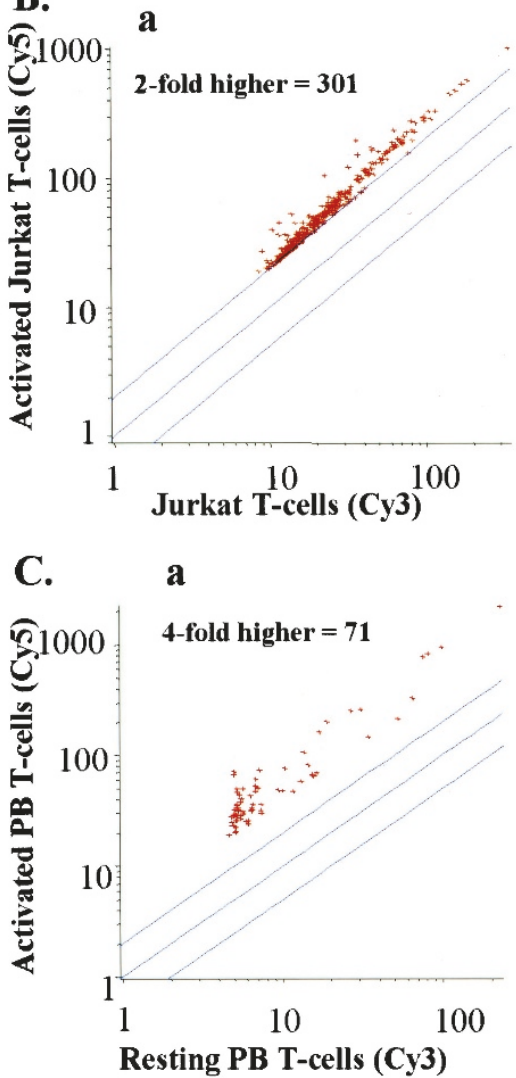

b

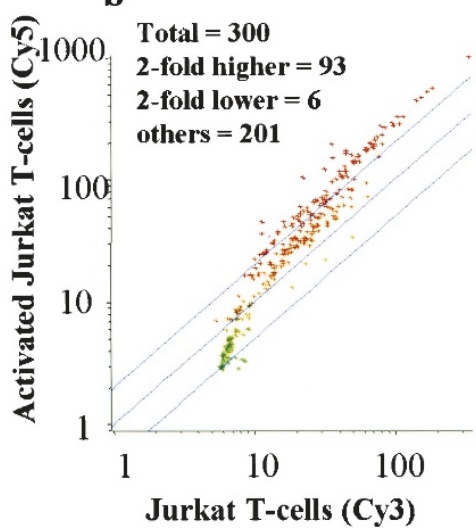

b
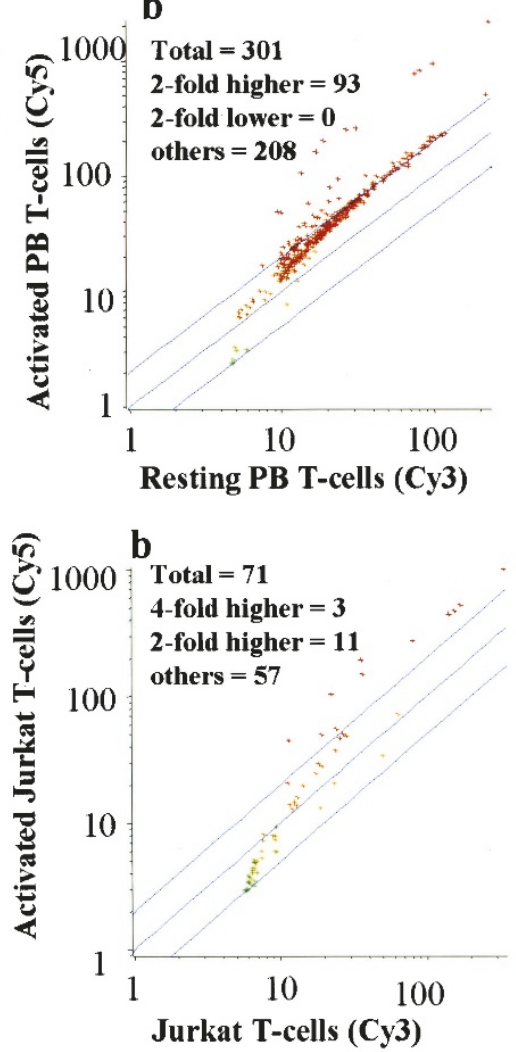

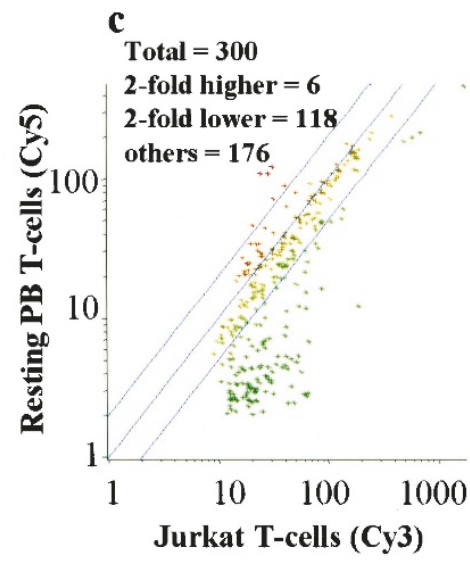

c
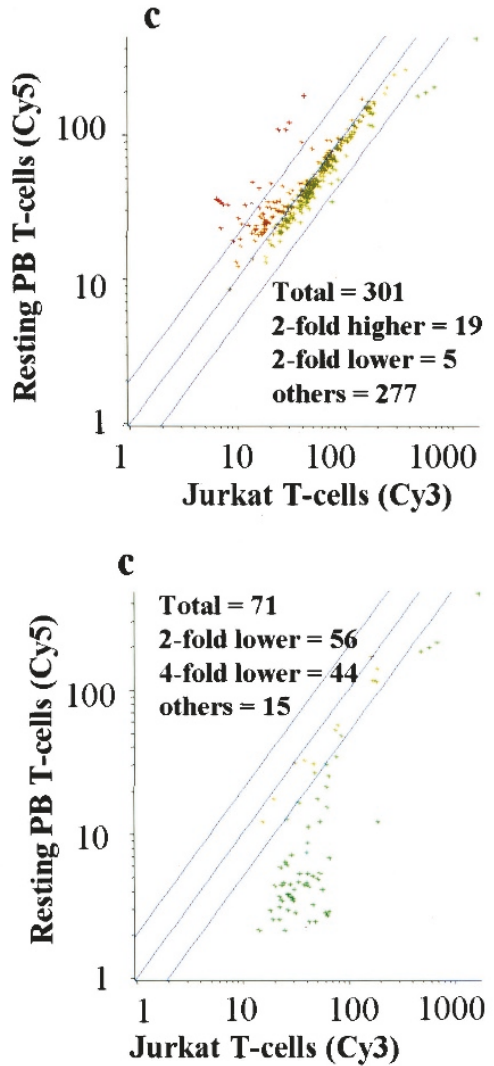

Figure 2.

Distribution of genes overexpressed greater than 2-fold during activation. A, Of the 6912 genes tested, 300 were overexpressed greater than 2-fold in PB T cells (a) Among these 300 overexpressed genes, 93 genes were also overexpressed in Jurkat T cells during activation (b), and 118 genes were expressed more than 2 -fold higher in nonactivated Jurkat than in resting PB T cells (c). B, Profile of 301 genes overexpressed more than 2-fold in Jurkat T cells (a) during activation, in PB T cells (b) during activation, and in resting PB T cells compared with nonactivated Jurkat T cells (c). C, Profile of 71 genes up-regulated by 4 -fold in PB T cells (a) during activation, in Jurkat T cells (b) during activation, and in resting PB T cells compared with nonactivated Jurkat T cells (c). Red points indicate overexpressed genes and green points represent underexpressed genes. Genes above the upper blue line were overexpressed by more than 2-fold, whereas genes below the lower blue line were underexpressed by more than 2-fold.

1998) to ultimately induce cell survival and inhibit apoptosis. The expression of transcription factors such as jun and fos are also activated by PI3-kinase and AKT (Duan et al, 2002; Walsh et al, 2002) and contribute to T-cell survival. Cross-linking of cell surface proteins such as CDW52 (CAMPATH-1 antigen), a glycopeptide antigen expressed on $\mathrm{T}$ and $\mathrm{B}$ lymphocytes, by anti-CD52 antibody mediates effective growth inhibition, supporting its important role in T-cell transformation (Rowan et al, 1998). Another cell surface antigen, endoglin (a recently recognized component of the transforming growth factor- $\beta$ receptor complex), is a strong marker of neovascularization and may thus play a role in Jurkat T-cell leukemogenesis (Calabro et al, 2003). Furthermore, several other genes in this list (Table 6) may be influenced by the persistent activation of the PI3-kinase pathway. For example, matrix metalloproteinase secretion in some cell types occurs via the PI3 kinase-AKT pathway (Ellerbroek et al, 2001). Matrix metalloproteinases are critical for cell 


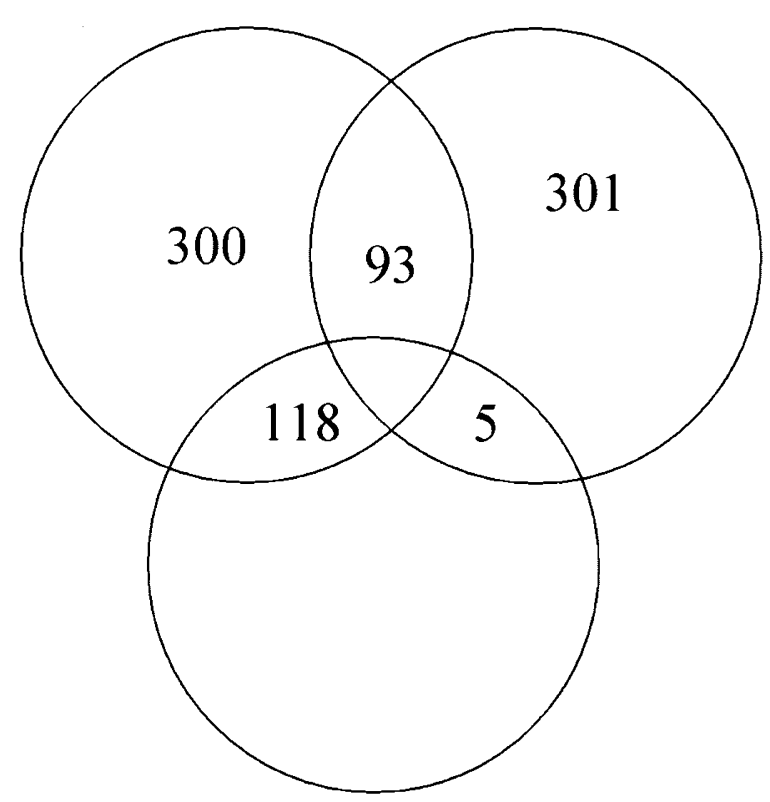

Figure 3.

A Venn diagram showing the overlaps of up-regulated genes during T-cell activation. Among the 300 genes that were up-regulated more than 2-fold (left circle) during PB T-cell activation, 93 of them were also overexpressed more than 2-fold during activation of Jurkat T cells (right circle). Approximately 118 genes (bottom circle) up-regulated during PB T cell activation were constitutively up-regulated in nonstimulated Jurkat T cells compared with resting PB T cells.

adhesive and invasive properties of malignant cells including leukemic T cells (Stetler-Stevenson et al, 1997) and may contribute to the neoplastic phenotype of Jurkat $T$ cells. Further functional studies of these differentially expressed genes may lead to identification of novel leukemia-specific genes.

\section{Validation of Gene Expression by Real-Time RT-PCR}

To validate the gene expression patterns observed in our microarray experiments, we used the same source of mRNAs to perform quantitative fluorescent realtime RT-PCR (Elenitoba-Johnson et al, 2002). The RT-PCR quantitation of nine selected gene transcripts (Table 7) showed that the trend of gene expression during activation of both Jurkat and PB T cells was consistent between the microarray and RT-PCR data. The overall correlation coefficient between relative expression levels from the microarray analysis and from RT-PCR was 0.81. Using this methodology, our previous validation of microarray data has shown approximately $70 \%$ concordance (Fillmore et al, 2002; Robetorye et al, 2002).

In summary, comparative microarray studies of PHA-induced activation of PB T cells and Jurkat cells reveal that genes associated with proliferation, protein synthesis, cytoskeletal construction, and signaling were similarly up-regulated, whereas genes associated with cell cycle inhibition and apoptosis were down-regulated at similar levels. A subset of genes induced during PB T-cell activation, however, were constitutively expressed at high levels in nonactivated
Jurkat cells, suggesting a role for these genes in the neoplastic transformation and/or biology of T-ALL. Finally, with the exception of a small subset of genes, the high degree of overlap between the gene expression profiles of the two cell types suggests that Jurkat $\mathrm{T}$ cells remain a suitable model for $\mathrm{T}$-cell activation.

\section{Materials and Methods \\ Isolation of Human PB T Cells}

Fresh PB was obtained from normal donors. To isolate T cells, buffy coat samples were collected from blood using Ficoll-Paque (Amersham Pharmacia, Piscataway, New Jersey), following the manufacturer's instructions. $\mathrm{CD}^{+} \mathrm{T}$ cells were then isolated by Dynal beads coated with CD2-specific mAbs (Dynal CD2 CELLection Kit; DYNAL Biotech, Oslo, Norway), following the manufacturer's protocol.

\section{T-Cell Activation}

Both $\mathrm{CD}^{+} \mathrm{T}$ cells and leukemic Jurkat $\mathrm{T}$ cells were cultured in RPMI-1640 (Life Technologies, Rockville, Maryland) containing $10 \%$ fetal bovine serum and activated in the same medium supplemented with $1 \%$ (v/v) PHA (Life Technologies, Carlsbad, California) for 24 hours at $37^{\circ} \mathrm{C}$ in $5 \% \mathrm{CO}_{2}$.

\section{Separation of Total RNA and mRNA}

Total RNA was extracted by using the TRIZol reagent (Life Technologies, Carlsbad, California). Messenger RNA was purified from total RNA using poly(T)-coated beads (Oligotex mRNA Isolation Kit; Qiagen, Valencia, California), according to the manufacturer's protocol.

\section{Preparation and Hybridization of Fluorescent-Labeled cDNA}

For each comparative array hybridization, fluorescent cDNA was synthesized by reverse transcription from a test sample (activated T-cell mRNA or activated Jurkat mRNA) in the presence of Cy5-dUTP and from a reference sample (nonactivated T-cell mRNA or Jurkat mRNA) with Cy3-dUTP, using the Superscript II RT kit (Life Technologies, Carlsbad, California) with several modifications. The RT reaction mixture contained $0.5 \mathrm{~mm}$ dATP, dCTP, and dGTP, $0.2 \mathrm{~mm}$ dTTP, and $0.1 \mathrm{~mm}$ Cy5- or Cy3-dUTP (Amersham Pharmacia). The labeling reaction was stopped by the addition of $5 \mu$ l of $500 \mathrm{~mm}$ EDTA. The mRNA was hydrolyzed by adding $10 \mu$ of $1 \mathrm{~N} \mathrm{NaOH}$ and heating to $65^{\circ} \mathrm{C}$ for 1 hour. The resulting alkaline solution was neutralized with $1 \mathrm{~m}$ Tris- $\mathrm{HCl}$ (pH 7.5). Unincorporated nucleotides were removed using a Biospin 6 column (Bio-Rad Laboratories, Hercules, California). The two fluorescently labeled cDNA samples were mixed, concentrated using a Microcon 30 microconcentrator (Amicon Inc., Beverly, Massachusetts), and hybridized to an arrayed slide (Research Genetics, Huntsville, Alabama) containing 6912 clones as described previously (Manos 
Table 6. Constitutively Overexpressed Genes and ESTs in Nontreated Jurkat T cells ${ }^{a}$ in Comparison to Nonactivated PB T Cells

\begin{tabular}{|c|c|}
\hline GenBank/Unigene & Description \\
\hline \multicolumn{2}{|c|}{ Cell growth and/or maintenance } \\
\hline Hs.153889 & Transforming growth factor- $\beta$ precursor \\
\hline Hs.2017 & Latent transforming growth factor- $\beta$ binding protein 1 \\
\hline Hs.2206 & TNF type 2 receptor-associated protein \\
\hline \multicolumn{2}{|c|}{ Transcription regulator } \\
\hline Hs.75889 & V-jun avian sarcoma virus 17 oncogene homolog \\
\hline Hs.83428 & $\begin{array}{l}\text { Nuclear factor of kappa light polypeptide gene enhancer in B } \\
\text { cells }\end{array}$ \\
\hline Hs.44450 & Sp3 transcription factor \\
\hline Hs.169465 & FOS-related antigen 1 \\
\hline \multicolumn{2}{|l|}{ Cellular } \\
\hline \multicolumn{2}{|l|}{ component } \\
\hline AA278759 & Proteoglycan 1 , secretory granule \\
\hline AA410265 & Lysosomal-associated multispanning membrane protein-5 \\
\hline Hs.1908 & Hematopoetic proteoglycan core protein \\
\hline \multicolumn{2}{|r|}{ 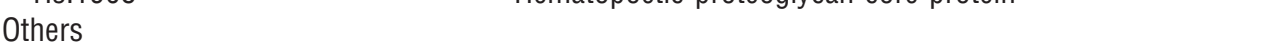 } \\
\hline R38270 & Homo sapiens clone 24734 mRNA sequence \\
\hline AA410517 & Protease inhibitor 6 (placental thrombin inhibitor) \\
\hline N73222 & ESTs, moderately similar to hypothetical protein ( $H$. sapiens) \\
\hline R38105 & ESTs \\
\hline H70017 & Mannosidase, alpha, class 2A, member 1 \\
\hline H17882 & Kallmann syndrome 1 sequence \\
\hline R47979 & Human HLA-DR $\alpha$-chain mRNA \\
\hline AA446108 & Endoglin \\
\hline Hs.2248 & Interferon $\gamma$-induced protein 10 \\
\hline Hs.75462 & Human BTG2 \\
\hline Hs.100000 & S100 calcium-binding protein A8 (calgranulin A) \\
\hline Hs.112405 & S100 calcium-binding protein A9 (calgranulin B) \\
\hline Hs.59847 & ESTs \\
\hline Hs.29748 & ESTs \\
\hline Hs.28102 & ESTs \\
\hline Hs.3452 & ESTs \\
\hline Hs. 151031 & Human Aac11 \\
\hline Hs.77961 & Major histocompatibility complex, class I, C \\
\hline Hs.159120 & CDW52 antigen (CAMPATH-1 antigen) \\
\hline Hs.3842 & Homo sapiens ATPase homolog \\
\hline Hs.10590 & ESTs \\
\hline Hs.155609 & CAMP-dependent protein kinase regulatory subunit type I \\
\hline Hs.15953 & ESTs \\
\hline Hs.76807 & HLA class II histocompatibility antigen \\
\hline Hs.21278 & ESTs \\
\hline Hs.180029 & Human guanine nucleotide exchange factor \\
\hline Hs.62954 & Enolase 1 (alpha) \\
\hline Hs.347 & Lactotransferrin \\
\hline Hs.155485 & Huntingtin-interacting protein 2 \\
\hline Hs.151738 & Matrix metalloproteinase 2 \\
\hline Hs.8249 & ESTs \\
\hline Hs.16291 & ESTs \\
\hline Hs.8248 & Ubiquinone oxidoreductase precursor \\
\hline Hs.13059 & ESTS \\
\hline R39288 & ESTs \\
\hline
\end{tabular}

${ }^{a}$ These genes were overexpressed greater than 4-fold during PB T-cell activation.

and Jones, 2001). Hybridization patterns were captured electronically using a two-color confocal laser microscope (Molecular Dynamics, Sunnyvale, California).

\section{Microarray Experimental Design and Data Analysis}

To directly analyze the changes in transcriptional profile induced by activation, mRNA from PHA- 
Table 7. Validation of Microarray Gene Expression Data by Real-Time RT-PCR

\begin{tabular}{lccccc}
\hline & \multicolumn{2}{c}{$\begin{array}{c}\text { Activated PB T cells vs resting T } \\
\text { cells }\end{array}$} & & \multicolumn{2}{c}{$\begin{array}{c}\text { Activated Jurkat vs nontreated } \\
\text { Jurkat T cells }\end{array}$} \\
\cline { 2 - 3 } \cline { 5 - 6 } Gene name & Microarray $^{a}$ & RT-PCR $^{a, b}$ & & Microarray $^{a}$ & RT-PCR $^{a, b}$ \\
\hline NFKB1 & 3.1 & 1.4 & & -1.5 & -3.1 \\
Fos B & 1.5 & 1.7 & -2.3 & -1.1 \\
Jun D & 1.6 & 1.7 & -2.0 & -1.2 \\
Jun B & & & & -3.9 \\
p16 & -1.5 & -3.5 & -1.9 & -1.7 \\
p27 & -2.2 & -1.5 & -3.0 & -1.3 \\
RPS14 & -1.8 & -2.2 & -2.1 & 1.7 \\
CDC 25A & 3.6 & 3.2 & & 1.8 & 1.5 \\
CD 68 & 3.6 & 3.5 & 1.6 & 1.8 \\
\hline
\end{tabular}

${ }^{a}$ The numbers represent the ratio of gene expression level between activated and nonactivated T cells

${ }^{b}$ Gene expression levels were normalized to the housekeeping gene GAPDH.

stimulated cells were hybridized directly with their nonactivated counterpart. As a control, Cy5-labeled cDNA from $\mathrm{CD}^{+}{ }^{+} \mathrm{T}$ cells was also hybridized with Cy3-cDNA from Jurkat cells. Each hybridization was performed in duplicate and repeated twice. Microarray analysis was performed using GeneSpring 4.0.4 (Silicon Genetics, Redwood City, California). Relative levels of transcripts between test and reference samples were shown as ratio of Cy5 signal (red fluorescence) versus Cy3 (green fluorescence) signal. Two-fold overexpression of a gene was indicated when the Cy5 signal intensity from the test sample was twice that of Cy3 (from the reference sample), and 2-fold underexpression was indicated by a Сy 3 signal intensity twice as high as that of Cy5. To ensure that the reported fold changes in gene expression were significant, $p<0.05$ was used as a cut-off value so that only those fold changes with $p$ values $<0.05$ were presented.

\section{Quantitative Fluorescent Real-Time RT-PCR}

RNA was reverse transcribed using the First-Strand cDNA Kit (Life Technologies, Carlsbad, California). After cDNA synthesis, $1 \mu$ of cDNA template was used for each PCR reaction. The forward and reverse primers used for PCR reactions were as follows: 5'CGACCACTTTGTCAAGCTCA-3' (forward, GAPDH), 5'-AGGGGAGATTCAGTGTGGTG-3' (reverse, GAPDH); 5'-CAAGGGTCTTGGGTCCTA-3' (forward, RPS 14), 5'-TGCCCTTGTGAACTGGTG-3' (reverse, RPS 14); 5'-GCACGACAACATCTCATTGG-3' (forward, NFKB1), 5'-TCCCAAGAGTCATCCAGGTC-3' (reverse, NFKB1); and 5'-TCTCTCAAGCTCGCCTCTTC-3' (forward, Jun B), 5'-ACGTGGTTCATCTTGTGCAG-3' (reverse, JunB). Real-time PCR was conducted using a LightCycler DNA Master SYBR Green I Kit (Roche Molecular Biochemicals, Indianapolis, Indiana), following the manufacturer's instructions with the following thermal cycling conditions: $95^{\circ} \mathrm{C}$ for 30 seconds, followed by 45 cycles of $95^{\circ} \mathrm{C}$ for 0 seconds, $55^{\circ} \mathrm{C}$ for 5 seconds, and $72^{\circ} \mathrm{C}$ for 10 seconds. Each gene was normalized to a housekeeping gene (GAPDH) before fold change was calculated based on the signal intensities at the midexponential stages of the real-time amplification curves.

\section{References}

Astoul E, Edmunds C, Cantrell DA, and Ward SG (2001). PI 3-K and T-cell activation: Limitations of T-leukemic cell lines as signaling models. Trends Immunol 22:490-496.

Black AC, Luo J, Chun S, and Tabibzadeh S (1997). Constitutive expression of the HTLV-I pX and env regions in Jurkat T-cells induces differential activation of SRE, CRE and NF kappa B pathways Virus Genes 15:105-117.

Boise LH, Minn AJ, June CH, Lindsten T, and Thompson CB (1995). Growth factors can enhance lymphocyte survival without committing the cell to undergo cell division. Proc Natl Acad Sci USA 92:5491-5495.

Calabro L, Fonsatti E, Bellomo G, Alonci A, Colizzi F, Sigalotti L, Altomonte M, Musolino C, and Maio M (2003). Differential levels of soluble endoglin (CD105) in myeloid malignancies. J Cell Physiol 194:171-175.

Chen H, Duncan IC, Bozorgchami H, and Lo SH (2002). Tensin 1 and a previously undocumented family member, tensin2, positively regulate cell migration. Proc Natl Acad Sci USA 99:733-738.

Chtanova T, Kemp RA, Sutherland AP, Ronchese F, and Mackay CR (2001). Gene microarrays reveal extensive differential gene expression in both cd4(+) and cd8(+) type 1 and type 2 T cells. J Immunol 167:3057-3063.

Cooper D and Pellis NR (1998). Suppressed PHA activation of $T$ lymphocytes in simulated microgravity is restored by direct activation of protein kinase C. J Leukoc Biol 63:550562.

Diehn M, Alizadeh AA, Rando OJ, Liu CL, Stankunas K, Botstein D, Crabtree GR, and Brown PO (2002). Genomic expression programs and the integration of the CD28 costimulatory signal in T cell activation. Proc Natl Acad Sci USA 99:11796-11801.

Duan R, Xie W, Li X, McDougal A, and Safe S (2002). Estrogen regulation of $\mathrm{c}$-fos gene expression through 
phosphatidylinositol-3-kinase-dependent activation of serum response factor in MCF-7 breast cancer cells. Biochem Biophys Res Commun 294:384-394.

Elenitoba-Johnson KS, Bohling SD, Jenson SD, Lin Z, Monnin KA, and Lim MS (2002). Fluorescence PCR quantification of cyclin d1 expression. J Mol Diagn 4:90-96.

Ellerbroek SM, Halbleib JM, Benavidez M, Warmka JK, Wattenberg EV, Stack MS, and Hudson LG (2001). Phosphatidylinositol 3-kinase activity in epidermal growth factorstimulated matrix metalloproteinase- 9 production and cell surface association. Cancer Res 61:1855-1861.

Ellisen LW, Palmer RE, Maki RG, Truong VB, Tamayo P, Oliner JD, and Haber DA (2001). Cascades of transcriptional induction during human lymphocyte activation. Eur $\mathrm{J}$ Cell Biol 80:321-328.

Feske S, Giltnane J, Dolmetsch R, Staudt LM, and Rao A (2001). Gene regulation mediated by calcium signals in $T$ lymphocytes. Nat Immunol 2:316-324.

Fillmore GC, Lin Z, Bohling SD, Robetorye RS, Kim CH, Jenson SD, Elenitoba-Johnson KS, and Lim MS (2002). Gene expression profiling of cell lines derived from T-cell malignancies. FEBS Lett 522:183-188.

Ghaffari-Tabrizi N, Bauer B, Villunger A, Baier-Bitterlich G, Altman A, Utermann G, Uberall F, and Baier G (1999). Protein kinase Ctheta, a selective upstream regulator of JNK/SAPK and IL-2 promoter activation in Jurkat T cells. Eur $\mathrm{J}$ Immunol 29:132-142.

Gonzales M and Bowden GT (2002). The role of PI 3-kinase in the UVB-induced expression of c-fos. Oncogene 21:27212728.

Kim G, Jun JB, and Elkon KB (2002). Necessary role of phosphatidylinositol 3-kinase in transforming growth factor beta-mediated activation of Akt in normal and rheumatoid arthritis synovial fibroblasts. Arthritis Rheum 46:1504-1511.

Klekotka PA, Santoro SA, Ho A, Dowdy SF, and Zutter MM (2001). Mammary epithelial cell-cycle progression via the alpha(2)beta(1) integrin: Unique and synergistic roles of the alpha(2) cytoplasmic domain. Am J Pathol 159:983-992.

Kreuzer T, Zarkovic N, Grube R, and Schaur RJ (1997). Inhibition of HeLa cell proliferation by 4-hydroxynonenal is associated with enhanced expression of the c-fos oncogene. Cancer Biother Radiopharm 12:131-136.

Lee YG, Macoska JA, Korenchuk S, and Pienta KJ (2002). MIM, a potential metastasis suppressor gene in bladder cancer. Neoplasia 4:291-294.

Lew DJ and Reed SI (1993). Morphogenesis in the yeast cell cycle: Regulation by Cdc28 and cyclins. J Cell Biol 120: 1305-1320.

Lindholm CK, Gylfe E, Zhang W, Samelson LE, and Welsh M (1999). Requirement of the Src homology 2 domain protein Shb for $T$ cell receptor-dependent activation of the interleukin-2 gene nuclear factor for activation of $T$ cells element in Jurkat T cells. J Biol Chem 274:28050-28057.

MacFarlane M, Cohen GM, and Dickens M (2000). JNK (c-Jun N-terminal kinase) and p38 activation in receptormediated and chemically-induced apoptosis of T-cells: Differential requirements for caspase activation. Biochem J 348 Pt 1:93-101.
Manos EJ and Jones DA (2001). Assessment of tumor necrosis factor receptor and Fas signaling pathways by transcriptional profiling. Cancer Res 61:433-438.

Morley SJ, Jeffrey I, Bushell M, Pain VM, and Clemens MJ (2000). Differential requirements for caspase-8 activity in the mechanism of phosphorylation of elF2alpha, cleavage of elF4GI and signaling events associated with the inhibition of protein synthesis in apoptotic Jurkat $T$ cells. FEBS Lett 477:229-236.

Natoli G, Costanzo A, Guido F, Moretti F, Bernardo A, Burgio VL, Agresti C, and Levrero M (1998). Nuclear factor kBindependent cytoprotective pathways originating at tumor necrosis factor receptor-associated factor 2. J Biol Chem 273:31262-31272.

Passegue E and Wagner EF (2000). JunB suppresses cell proliferation by transcriptional activation of p16(INK4a) expression. EMBO J 19:2969-2979.

Polyak $\mathrm{K}$, Lee $\mathrm{MH}$, Erdjument-Bromage $\mathrm{H}$, Koff $\mathrm{A}$, Roberts JM, Tempst $P$, and Massague J (1994). Cloning of p27Kip1, a cyclin-dependent kinase inhibitor and a potential mediator of extracellular antimitogenic signals. Cell 78:59-66.

Robetorye RS, Bohling SD, Morgan JW, Fillmore GC, Lim MS, and Elenitoba-Johnson KS (2002). Microarray analysis of B-cell lymphoma cell lines with the $t(14 ; 18)$. J Mol Diagn 4:123-136

Rowan W, Tite J, Topley P, and Brett SJ (1998). Cross-linking of the CAMPATH-1 antigen (CD52) mediates growth inhibition in human B- and T-lymphoma cell lines, and subsequent emergence of CD52-deficient cells. Immunology 95:427-436.

Rowan WC, Hale G, Tite JP, and Brett SJ (1995). Crosslinking of the CAMPATH-1 antigen (CD52) triggers activation of normal human T lymphocytes. Int Immunol 7:69-77.

Rudd CE (1999). Adaptors and molecular scaffolds in immune cell signaling. Cell 96:5-8.

Ruiz P, Coleman L, Wang F, and Enten J (2001). Differential kinetics of intracellular caspase-1-like and caspase-3-like enzyme activity in human alloreactive CD4(+) and CD8(+) T cells undergoing apoptosis. Clin Immunol 98:308-312.

Sawada N, Kojima T, Obata H, Saitoh M, Isomura H, Kokai Y, Satoh M, and Mori M (1996). P21waf-1/cip-1/sdi-1 is expressed at G1 phase in primary culture of hepatocytes from old rats, presumably preventing the cells from entering the $S$ phase of the cell cycle. Biochem Biophys Res Commun 228:819-824.

Schena M, Shalon D, Davis RW, and Brown PO (1995). Quantitative monitoring of gene expression patterns with a complementary DNA microarray. Science 270:467-470.

Stetler-Stevenson M, Mansoor A, Lim M, Fukushima P, Kehrl J, Marti G, Ptaszynski K, Wang J, and Stetler-Stevenson WG (1997). Expression of matrix metalloproteinases and tissue inhibitors of metalloproteinases in reactive and neoplastic lymphoid cells. Blood 89:1708-1715.

Sylvester AM, Chen D, Krasinski K, and Andres V (1998). Role of $\mathrm{c}-\mathrm{fos}$ and $\mathrm{E} 2 \mathrm{~F}$ in the induction of cyclin $\mathrm{A}$ transcription and vascular smooth muscle cell proliferation. J Clin Invest 101: 940-948

Takahashi A, Kono K, Amemiya $\mathrm{H}$, lizuka $\mathrm{H}$, Fujii $\mathrm{H}$, and Matsumoto $Y$ (2001). Elevated caspase-3 activity in peripheral blood $\mathrm{T}$ cells coexists with increased degree of T-cell apoptosis and down-regulation of TCR zeta molecules in patients with gastric cancer. Clin Cancer Res 7:74-80. 
Tanaka N, Abe H, Yagita H, Okumura K, Nakamura M, and Sugamura K (1997). Itk, a T cell-specific tyrosine kinase, is required for CD2-mediated interleukin-2 promoter activation in the human T cell line Jurkat. Eur J Immunol 27:834-841.

Teague TK, Hildeman D, Kedl RM, Mitchell T, Rees W, Schaefer BC, Bender J, Kappler J, and Marrack P (1999). Activation changes the spectrum but not the diversity of genes expressed by T cells. Proc Natl Acad Sci USA 96: 12691-12696.

Tsuchida M, Manthei ER, Alam T, Knechtle SJ, and Hamawy MM (1999). T cell activation up-regulates the expression of the focal adhesion kinase Pyk2: Opposing roles for the activation of protein kinase $\mathrm{C}$ and the increase in intracellular $\mathrm{Ca}^{2+}$. J Immunol 163:6640-6650.

Varghese J, Radhika G, and Sarin A (2001). The role of calpain in caspase activation during etoposide induced apoptosis in T cells. Eur J Immunol 31:2035-2041.

Vella AT, Dow S, Potter TA, Kappler J, and Marrack P (1998). Cytokine-induced survival of activated $T$ cells in vitro and in vivo. Proc Natl Acad Sci USA 95:3810-3815.

Walsh PT, Smith LM, and O'Connor R (2002). Insulin-like growth factor-1 activates Akt and Jun N-terminal kinases (JNKs) in promoting the survival of T lymphocytes. Immunology 107:461-471.
Wang LD, Hoeltzel M, Butler K, Hare B, Todisco A, Wang M, and Del Valle J (1997). Activation of the human histamine H2 receptor is linked to cell proliferation and c-fos gene transcription. Am J Physiol 273:C2037-C2045.

Welsh CF and Assoian RK (2000). A growing role for Rho family GTPases as intermediaries in growth factor- and adhesion-dependent cell cycle progression. Biochim Biophys Acta 1471:M21-M29.

Whisler RL, Liu B, Wu LC, and Chen M (1993). Reduced activation of transcriptional factor AP-1 among peripheral blood $\mathrm{T}$ cells from elderly humans after PHA stimulation: Restorative effect of phorbol diesters. Cell Immunol 152:96109.

Whisler RL, Newhouse YG, Beiqing L, Karanfilov BK, Goyette MA, and Hackshaw KV (1994). Regulation of protein kinase enzymatic activity in Jurkat $T$ cells during oxidative stress uncoupled from protein tyrosine kinases: Role of oxidative changes in protein kinase activation requirements and generation of second messengers. Lymphokine Cytokine Res 13:399-410. 